\title{
Basosquamous Cell Carcinoma of the Lower Lip Arising from Actinic Cheilitis: Case Report and up Date
}

\author{
Ana Maria de Oliveria Miranda, Thiago de Miranda Ferrari, Daniel Cohen Goldemberg, \\ Luciana Pantaleão, Andrea Pires, Eliane Pedra Dias \\ Department of Pathology, Medicine school, Federal Fluminense University, Rio de Janeiro, Brazil. \\ Email: anamiranda3@hotmail.com
}

Received September $2^{\text {nd }}, 2013$; revised October $3^{\text {rd }}$, 2013; accepted November $1^{\text {st }}, 2013$

Copyright (c) 2013 Ana Maria de Oliveria Miranda et al. This is an open access article distributed under the Creative Commons Attribution License, which permits unrestricted use, distribution, and reproduction in any medium, provided the original work is properly cited. In accordance of the Creative Commons Attribution License all Copyrights (C) 2013 are reserved for SCIRP and the owner of the intellectual property Ana Maria de Oliveria Miranda et al. All Copyright (C) 2013 are guarded by law and by SCIRP as a guardian.

\begin{abstract}
Background: Basosquamous carcinoma (BSC) is a rare non-melanoma skin cancer, considered to be a subtype of basal cell carcinoma (BCC). BSC often produces distant metastases with a higher risk of recurrence than that of BCC which is not commonly found in the lip. Case Report: A 57-year-old white female patient presented an ulcer on her lower lip that had an ongoing development for over six months. Physical examination, photo documentation, videoroscopy, scraped cytology, toluidine blue test, and biopsy of the ulcer were carried out. Results: Upon physical examination we observed an actinic cheilitis associated with the ulcer. Videoroscopy revealed the presence of fissures and erosion that had not been seen by oroscopy. Toluidine blue test was only positive for the region of the ulcer. Cytological analysis revealed rare nests compatible with carcinoma. Histopathology of the biopsy revealed a carcinoma with nests lined by basal cells associated with areas of squamous differentiation. The patient was then referred to surgery for the removal of the BCC. Analysis of the specimen showed free surgical margins and the immunohistochemical panel did not confirm the initial diagnosis of BCC, indicating a subtype of BSC. After surgery, the patient has been followed by periodic consultations. She is well and without further complications. Coments: BSC is considered to be an aggressive and rare tumor affecting mainly upper face and primarily affects men over 60 years of age. Since our patient is a woman presenting the lesion in the lower lip, this highlights the unusual and interesting presentation of this case report.
\end{abstract}

Keywords: Basosquamous Carcinoma; Lip; Actinic Cheilitis; Basal Cell Carcinoma

\section{Introduction}

Malignant labial neoplasms account for approximately $30 \%$ of all tumours occurring in the oral cavity including the lips. Of these $95 \%$ are classified as squamous cell carcinomas (SCC). The factors that influence the development of lip SCC include the use of tobacco and alcohol, chronic exposure to sunlight and individual susceptibility such as skin and eye color [1].

Basal cell carcinoma (BCC) is the most common malignant skin tumour worldwide, affecting mainly the head and neck region, most frequently occurring in upper two thirds of face. Like SCC, it presents many variant forms, which need to be correctly diagnosed [2]. Basosquamous carcinoma (BSC) or metatypical carcinoma [2,5-8] is one of the subtypes of BCC. It is a rare non-melanoma skin cancer, which often produces distant metastasis and presents a high recurrence risk [3,4].

Although the World Health Organization (WHO) and most researchers consider BSC as an aggressive subtype of BCC $[2,5,9]$, other authors classify BSC as a separate entity, causing controversy in the literature. Those that classify BSC as a different entity propose that this tumor contains areas characteristic of both BCC and SCC with no transition zone between them and with a propensity for distant metastases thus making it different from BCC or SCC once these tumors rarely metastasize $[3,6,7,10]$.

Despite classification controversy, it is generally accepted that BSC is a rare skin tumor ( $1 \%$ to $2 \%$ of all skin cancers), with higher local aggressiveness and a tendency to generate distant metastasis, highlighting the importance of a correct diagnosis [11]. Its location is 
mainly in head and neck, with only $1 \%$ appearing as labial lesions [12]. In view of its clinical similarities with BCC, it is only possible to diagnose BSC by performing an incisional biopsy associated with histopathological examination. Care should be given to the size of the biopsy once the histological features of BSC may be absent if tissue biopsy is too small [13]. With regards to histopathological features, this tumor presents areas typical of both BCC and SCC with the presence of basaloid and squamoid cells respectively. A transition zone with intermediate cells is evident between the area of BCC and SCC tumor cells [3]. The most commonly used antibody to differentiate basaloid SCC from BSC, is BerEP4 as the latter presents BerEP4 immunopositivity in contrast to the former [14]. Brantsch et al. [6] accept the use of BSC term for tumors which are similar to BCCs that have areas of squamous differentiation presenting positivity for Ber-EP4 associated with a negative result of epithelial membrane antigen (EMA).

The recommended treatment for BSC is the total removal of the tumour with a safety margin and a long term follow-up. For high-risk lesions (greater than $2 \mathrm{~cm}$ in diameter) and those with lymphatic and perineural invasion, radiotherapy and sentinel node biopsy should be considered [10]. A recent publication claiming to be the first European survey on different epidermal tumours treated by Mohs Micrographic Surgery (MMS) has pointed out the dangers that BSC poses if not properly managed. BSC seems to present a higher recurrence rate when compared to BCC, with an aggressive growth pattern, even when compared to SCC and treated by MMS [15], reinforcing the need for a wide surgical margin of tumors and close follow-up of these patients.

Actinic cheilitis (AC) is a potentially malignant disorder that gives rise to $95 \%$ of carcinomas of the lip and is associated with sun exposure, affecting mostly white men over 50 years of age $[16,17]$. Histologically, the most frequent findings of AC are solar elastosis associated with various degrees of dysplasia and inflammation [18]. The objective of this report is to present an unusual case of basosquamous carcinoma on the lower lip associated with actinic cheilitis.

\section{Case Report}

A 57-year-old, alcoholic, non-smoker, white female patient, came July 2010, to the Oral Medicine Outpatient Facility at the Antonio Pedro University Hospital, School of Medicine, UFF, Niterói, RJ, due to the development of an ulcer on her lower lip. The ulcer evolved over six months at the site of a recurrent herpes labialis (Figure 1(A)).

During medical history, although the patient reported that she had not worked in activities that involved excessive sun exposure; it was common for her to be

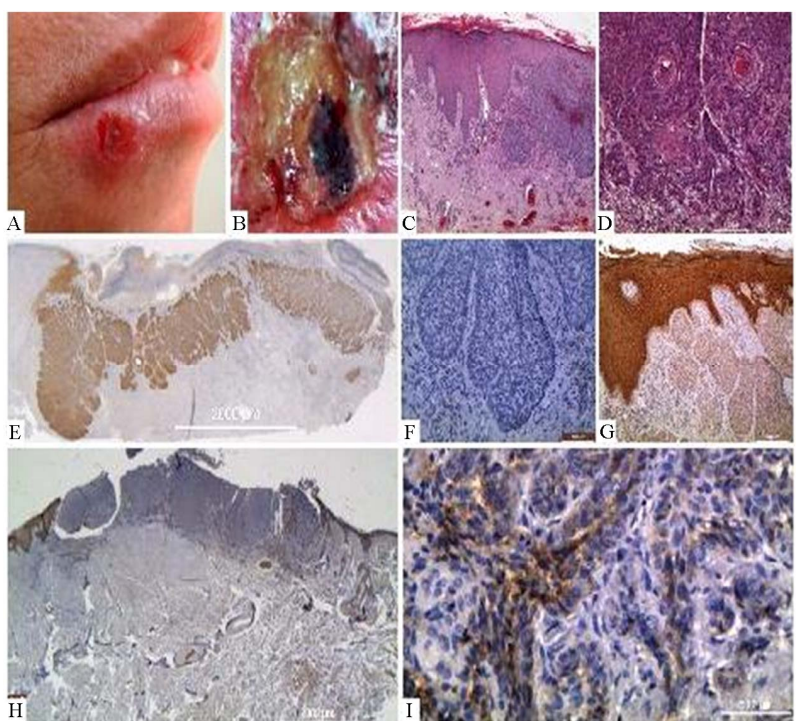

Figure 1. Basosquamous cell carcinoma: (A) clinical aspects; (B) videoroscopy of ulcer; (C), (D) Histopathological aspects by hematoxilin-eosin; (E) immunostaining with CK-17 antibody, positive; (F) EMA antibody, negative; (G), (H) CK16 antibody, negative; Ber-EP4 antibody, positive.

exposed to sunlight during leisure moments. She was born and lives in a town by the seaside, and when she was younger, she used to go to the beach, every weekend in summer, being exposed to the sun approximately 4 hours per day for more than 15 years with no protection of any kind of sunscreen. The patient underwent physical examination, photo documentation, videoroscopy (Figure 1(B)), scraped cytology, toluidine blue test, and biopsy.

During physical examination the lower lip presented an ulcer with spontaneous bleeding measuring $0.8 \times 0.8$ $\mathrm{cm}$ located partially on the lip and partially on the skin. The surrounding vermilion zone of the lip was dry, hardened and flaky presenting oedema and the blurring of the vermilion border leading to the initial clinical diagnosis of a severe actinic cheilitis, with the possibility of a SCC at the ulceration site. Crust, erythema, pigmented blemish and white plaques, were also observed during physical examination. No intra-oral lesions were observed.

Videoroscopy was performed with MAXX digital microscope (AVANTSCOPE MAXX ${ }^{\mathrm{TM}}$ ), with a magnification factor of 50 times (Figure 1(B)) revealing lesions with the presence of fissures and erosion with a positive toluidine blue test. Cytological analysis revealed rare nests of compatible with carcinoma. Histopathology of the directed biopsy fragment revealed presence of solar elastosis and a carcinoma with nests lined with basal cells, with areas of squamous differentiation (Figures 1(C) and (D)). Immunohistochemistry showed immunoreactivity to anti-CK17 and anti-CK 16 (Figures 1(E), (G) and (H)). 
After being submitted to all tests the patient was referred for removal of the BCC in October 2010. Analysis of the specimen from surgical fragment showed free surgical margins and the same histological aspects as was described for the incisional biopsy. We expanded the immunohistochemical panel, using epithelial membrane antigen (EMA) and BerEP4, which confirmed the diagnosis of BSC subtype and not BCC (Figures 1(F) and (I)). After surgery the lesion site showed good healing. During the last two years the patient has been followed up by periodic consultations every 6 months and until now she is well with no further changes, interestingly without showing new herpes labialis episodes.

\section{Comments}

BSC is a controversial rare aggressive skin tumour. It is characterized in some studies as a variant of BCC with squamous differentiation while in others it is considered as an independent tumour. Although there is a high risk of recurrence and one of its characteristics is its capacity to metastasize, giving rise both to local and distant metastasis, this is not a rule $[2,6,19]$. Some authors believe that sentinel lymph node biopsy could be useful for suggested high-risk BCC, although the routine use of SLNB for BCC is not recommended [20].

Previous studies report that BSC is predominant in fair-skinned males over sixty years of age, affecting areas of higher sunlight exposure, such as the head and neck [7]. Martin et al. [10] evaluated 31 cases of BSC in 28 patients with an average age of 68 years. Of these only $25 \%$ were female patients. Their results confirm the main trend in the literature finding BSC most often (75\%) in head and neck region. Similar results were obtained by Tarallo et al. [7] who found twice the number of men with BSC when compared to female patients, with an average age of 70 years. Leibovitch et al. [12] performed a larger study involving 178 cases of BSC with a mean age of 63 years. Of these approximately 2/3 (115 cases) occur in men and 1/3 (63) in women. The patient reported in this case is slightly below sixty years of age, matching the suggested most prevalent age of onset by most studies. Although, the patient is female, while most BSCs occur in male patients, her fair skin is consistent with the majority of cases reported in the literature.

Garcia et al. [20] performed a recent review of the literature report that a variety of studies have revealed that within the head and neck the most affected region is, the central area of the face and in particular the perinasal region. In accordance, after evaluating 35 patients with BSC, Borel [21] showed that the head and neck, particularly the central region of face, correspond to the area that is subjected to higher sunlight exposure. Yet in another study Leibovitch et al. [13] found the same results showing that the main location of BSC was the nas- al region (33\%), followed by the ear (18.5\%) and the periocular area (11.2\%), while the lip was affected in only $1 \%$ of the cases.

The case reported here aroused interest in its unusual localization - the patient's lower lip-and its low frequency especially in women given that lesions in this particular region represent only $1 \%$ of BSC cases reported in previous studies. Two reviews published in the 1970 s report that a frequency of 1 to $2 \%$ of non melanoma skin cancers is BSC. Schuller et al. [11] investigated 2565 cases of non-melanoma lesions of skin and identified 33 cases of BSC (1.2\%) while Borel [21] performed a review of the literature showing that among 1706 cases of non-melanoma cancers reported, 35 (2\%) cases were BSC. In a more recent study (2011) performed by Tarallo et al. [7], this incidence has grown to approximately 5\%.

BSC has considerable pathological relevance because of its local aggressiveness, risk of distant metastasis mainly affecting the lungs, and its increased risk of recurrence. Bowman et al. [3] reported a prevalence of metastasis of $7.4 \%$ for BSC, which was significantly higher than the prevalence of metastasis $0.87 \%$ for SCC and concluded that tissue invasion displayed by BSCs is similar to that of BCC or SCC but with a higher frequency of pulmonary metastasis than SCC.

Borel's [21] studies showed that $47 \%$ of the 35 patients with BSC presenting recurrent lesions even after treatment with a wide surgical excision. Martin et al. [10] evaluated 31 cases of BSC in 28 patients, observing the presence of recurrent tumours in 9 (32\%) patients after treatment, lymph node metastasis in five (17\%) patients, and one (4\%) patient developing lung metastasis. In their study, they concluded that the prognostic factors for recurrence were positive surgical margins, male gender, lymphatic invasion, perineural invasion and although they reported that tumor size was not statistically significant, all cases of lymphatic metastasis had tumours larger than $2 \mathrm{~cm}$.

As there are no clinical aspects that can distinguish BSC from BCC, diagnosis is based on its histopathological features. But Giacomel et al. [22] suggest that dermoscopy can be used to provide additional morphological information, as it was used in this study. This tumour has been poorly defined in the dermatological literature with confusing clinical and histological results, and in many studies not mentioning or recognizing the lesion [2,7,20]. Brantsch et al. [6] claim that immunohistochemistry distinguishes BSC from BCC and SCC. BSC is a similar to BCC in that it shows areas of squamous differentiation with immunoreactivity to BerEP4 and immunonegativity to EMA. Histopathological examination of the patient reported in this paper revealed carcinoma with tumor nests lined by basal cells, areas of 
squamous differentiation, positivity for BerEP4 and negativity for EMA, exactly as described in papers concerning BSC description [14].

Although rare in women and in the lower lip, this case alerts us to the possibility of tumors other than SCC affecting lips. This reinforces the need to investigate ulcers associated or not associated with actinic cheilitis, by cytopathological and histopathological examination. BSC, although rare and nonspecific clinical presentation, requires an accurate diagnosis because of its frequency of recurrence and greatly increased incidence of pulmonary metastasis compared to BCC and SCC.

\section{REFERENCES}

[1] A. Antunes and A. Antunes, "Estudo Retrospectivo e Revisão de Literatura dos Tumores dos Lábios: Experiência de 28 Anos,” Revista Brasileira de Cancerologia, Vol. 50, No. 4, 2004, pp. 295-300.

[2] C. Gregoire, D. Adler, S. Madey and R. B. Bell, "Basosquamous Carcinoma Involving the Anterior Skull Base: A Neglected Tumor Treated Using Intraoperative Navigation as A Guide to Achieve Safe Resection Margins," Journal of Oral and Maxillofacial Surgery, Vol. 69, No. 1, 2011, pp. 230-236.

[3] P. LeBoit, "Pathology and Genetics of Skin Tumours," World Health Organization, 2006.

[4] K. Brantsch, K. Sotlar, C. Brod and H. Breuninger, "Metastatic Basosquamous Carcinoma: Report of Two Cases,” Dermatologic Surgery, Vol. 34, No. 12, 2008, pp. 1738-1741.

[5] M. Tarallo, E. Cigna, R. Frati, S. Delfino, D. Innocenzi, U. Fama, et al., "Metatypical Basal Cell Carcinoma: A Clinical Review,” Journal of Experimental \& Clinical Cancer Research, Vol. 27, 2008, p. 65. http://dx.doi.org/10.1186/1756-9966-27-65

[6] N. Sendur, G. Karaman, E. Dikicioglu, C. Z. Karaman and E. Savk, "Cutaneous Basosquamous Carcinoma Infiltrating Cerebral Tissue,” Journal of the European Academy of Dermatology and Venereology, Vol. 18, No. 3, 2004, pp. 334-336.

[7] P. H. Bowman, J. L. Ratz, T. G. Knoepp, C. J. Barnes and E. M. Finley, "Basosquamous Carcinoma," Dermatologic Surgery, Vol. 29, No. 8, 2003, pp. 830-832.

[8] A. H. M. M. Arits, M. H. J. Schlangen, P. J. Nelemans and N. W. J. Kelleners-Smeets, "Trends in the Incidence of Basal Cell Carcinoma by Histopathological Subtype,” Journal of the European Academy of Dermatology and Venereology, Vol. 25, No. 5, 2011, pp. 565-569.

[9] D.-S. Jung, H.-H. Cho, H.-C. Ko, Y.-C. Bae, C.-K. Oh, M.-B. Kim, et al., "Recurrent Basal Cell Carcinoma Following Ablative Laser Procedures," Journal of the American Academy of Dermatology, Vol. 64, No. 4, 2011, pp. 723-729.

[10] R. C. G. Martin, M. J. Edwards, T. G. Cawte, C. L. Se- well and K. M. McMasters, "Basosquamous Carcinoma," Cancer, Vol. 88, No. 6, 2000, pp. 1365-1369. http://dx.doi.org/10.1002/(SICI)1097-0142(20000315)88: 6<1365::AID-CNCR13>3.0.CO, Vol. 2-Y

[11] D. E. Schuller, J. W. Berg, G. Sherman and C. J. Krause, "Cutaneous Basosquamous Carcinoma of the Head and Neck: A Comparative Analysis,” Otolaryngology—Head and Neck Surgery, Vol. 87, No. 4, 1979, pp. 420-427.

[12] I. Leibovitch, S. C. Huilgol, D. Selva, S. Richards and R. Paver, "Basosquamous Carcinoma: Treatment with Mohs Micrographic Surgery,” Cancer, Vol. 104, No. 1, 2005, pp. 170-175.

[13] M. Asuquo, O. Otei, C. Agbor, S. Ogbudu, G. Ebughe and J. Omotoso, "Basosquamous Carcinoma: Florid Lesion in a Nigerian,” African Journal of Cancer, Vol. 2, No. 1, 2010, pp. 41-43. http://dx.doi.org/10.1007/s12558-010-0065-5

[14] T. W. Beer, P. Shepherd and J. M. Theaker, "Ber EP4 and Epithelial Membrane Antigen Aid Distinction of Basal Cell, Squamous Cell and Basosquamous Carcinomas of the Skin,” Histopathology, Vol. 37, No. 3, 2000, pp. 218223. http://dx.doi.org/10.1046/j.1365-2559.2000.00999.x

[15] A. M. Skaria, "Recurrence of Basosquamous Carcinoma after Mohs Micrographic Surgery,” Dermatology, Vol. 221, No. 4, 2010, pp. 352-355. http://dx.doi.org/10.1159/000320127

[16] A. Markopoulos, E. Albanidou-Farmaki and I. Kayavis, "Actinic Cheilitis: Clinical and Pathologic Characteristics in 65 Cases,” Oral Diseases, Vol. 10, No. 4, 2004, pp. 212-216.

[17] A. M. O. Miranda, L. G. Soares, T. M. Ferrari, D. G. Silva, M. E. V. Falabella and E. M. B. Tinoco, "Prevalence of Actinic Cheilitis in a Population of Agricultural Sugarcane Workers," Acta Odontológica Latinoamericana, Vol. 25, No. 2, 2012, pp. 201-206.

[18] A. S. R. Cavalcante, A. L. Anbinder and Y. R. Carvalho, "Actinic Cheilitis: Clinical and Histological Features," Journal of Oral and Maxillofacial Surgery, Vol. 66, No. 3, 2008, pp. 498-503.

[19] H. M. Chedid, A. D. S. Menezes, K. F. Aikawa, C. N. Lehn, A. Rapoport, A. M. da C. Mercante, et al., "Neck Skin Collision Tumor," Revista do Colégio Brasileiro de Cirurgiões, Vol. 38, No. 1, 2011, pp. 66-70.

[20] Y. Yoshida, T. Shiomi, M. Tahira and O. Yamamoto, "Metastatic Basosquamous Carcinoma Detected by Sentinel Lymph Node Biopsy,” Journal of Dermatology, Vol. 40, No. 8, 2013, pp. 635-637.

[21] C. Garcia, E. Poletti and A. N. Crowson, "Basosquamous Carcinoma," Journal of the American Academy of Dermatology, Vol. 60, No. 1, 2009, pp. 137-143.

[22] J. Giacomel, A. Lallas, G. Argenziano, C. Reggiani, S. Piana, Z. Apalla, et al., "Dermoscopy of Basosquamous Carcinoma,” British Journal of Dermatology, Vol. 169, No. 2, 2013, pp. 358-364. 